\title{
Signature of light sterile neutrinos at IceCube
}

\author{
Bhavesh Chauhan \\ Physical Research Laboratory, Ahmedabad 380009, India \\ and Indian Institute of Technology Gandhinagar, Palaj 382355, Gandhinagar, India \\ Subhendra Mohanty ${ }^{\dagger}$ \\ Physical Research Laboratory, Ahmedabad 380009, India
}

(Received 30 August 2018; published 25 October 2018)

\begin{abstract}
The MiniBooNE collaboration has recently reported evidence for a light sterile neutrino with large mixing angles, thus corroborating the measurement by LSND twenty years ago. Such a state would be directly in conflict with Planck measurement of big bang nucleosynthesis $N_{\text {eff }}$ unless there is selfinteraction in the sterile sector. Our objective is to investigate if such interactions could result in resonant absorption in the cosmogenic neutrino spectrum and its consequences for the IceCube experiment. We show that it is possible to give independent bounds on sterile neutrino parameter space from IceCube observations with the dips in the spectrum corresponding to the neutrino masses.
\end{abstract}

DOI: $10.1103 /$ PhysRevD.98.083021

\section{INTRODUCTION}

The MiniBooNE collaboration has recently reported excess in the electron neutrino and antineutrino appearance channels that is consistent with the sterile neutrino hypothesis [1]. The best-fit point,

$$
\Delta m_{41}^{2}=0.041 \mathrm{eV}^{2} \quad \text { and } \quad \sin ^{2}\left(2 \theta_{\mu e}\right)=0.958
$$

is consistent with the earlier measurements by the LSND collaboration [2]. In fact, the combined significance of the two data sets is $6.1 \sigma$. These results, however, are in tension with data from disappearance experiments like MINOS+ and IceCube. Other experiments like KARMEN and OPERA have not been able to confirm this excess, but they do not rule it out completely either [3].

The existence of such light states with large mixing angles is also in conflict with cosmology. The Planck measurement cosmic microwave background (CMB) anisotropy puts severe constraints on the number of thermalized relativistic degrees of freedom $\left(N_{\text {eff }}\right)$ around the epoch of big bang nucleosynthesis (BBN), i.e., $T_{\gamma}=$ $1 \mathrm{MeV}$ [4]. One possible resolution to this puzzle is to assume self-interactions in the sterile sector [5-10].

\footnotetext{
bhavesh@prl.res.in

†mohanty@prl.res.in
}

Published by the American Physical Society under the terms of the Creative Commons Attribution 4.0 International license. Further distribution of this work must maintain attribution to the author(s) and the published article's title, journal citation, and DOI. Funded by SCOAP ${ }^{3}$.
Because of the large thermal effective potential, the mixing between sterile and active neutrino is suppressed in the early universe but is allowed to be large today. Hence the sterile neutrinos are produced efficiently only at low temperatures after recoupling [11]. This provides a very strong constraint that the $T_{\text {rec }}<1 \mathrm{MeV}$, which rules out small gauge couplings in the sterile sector [6]. Because of mixing, the lighter neutrinos also interact with the new gauge boson, which affects their free streaming in the early universe, which is constrained from CMB $[12,13]$. It was recently pointed out that taking constraints from $\sum m_{\nu}$ rules out any viable parameter space for $m_{s}>0.2 \mathrm{eV} \mathrm{[14].}$ However, the authors also propose several scenarios that weaken these new constraints. For gauge coupling in the range $0.1-1$, one requires a gauge boson of mass $10-50 \mathrm{MeV}$ to reconcile sterile neutrinos with cosmology. Moreover, such interactions can also be mediators to dark matter, which can simultaneously solve the small-scale crisis of $\Lambda \mathrm{CDM}[10,15,16]$.

It was shown in [17] that MeV scale secret interaction of neutrinos gives rise to absorption lines in the very high energy neutrino spectrum. Such lines can be seen by neutrino telescopes like IceCube. The IceCube HESE data have featured a prominent gap in the spectrum for neutrino energies in the range 400-800 $\mathrm{TeV}$ [18-20]. In the past, several authors have tried to explain this gap using resonant absorption in well-motivated models such as $\nu 2 \mathrm{HDM}$ [17] and gauged $U(1)_{L_{\mu}-L_{\tau}}$ [21]. Recently it was also proposed that one can explain the absence of Glashow resonance using t-channel resonant absorption [22]. All these explanations assume a flavor-universal single power law flux for incoming neutrinos. The IceCube data can also be 
explained by decaying dark matter [23-28], leptoquark like states [29-33], and by modifying assumptions of the source. The leptoquark explanation is highly constrained from LHC data [33-35].

In this paper, we look at resonant absorption of cosmogenic neutrinos from both cosmic neutrino and sterile neutrino background. In Sec. II we describe the model for sterile neutrino with self-interactions. In Sec. III, we discuss the basics of neutrino absorption and explain a few benchmark scenarios. In Sec. IV we look at the six-year IceCube data and provide some constraints on the model. We also provide the parameter space favored by IceCube independent of other short baseline experiments. In Sec. V we provide the results and discuss certain aspects of the analysis before we conclude.

\section{MODEL DESCRIPTION AND COSMOLOGICAL CONSTRAINTS}

To accommodate light sterile neutrino with cosmology, we extend the standard model by introducing a left-handed sterile neutrino $\left(\nu_{s}\right)$, which is charged under an additional gauge symmetry $U(1)_{X}$. The new gauge boson $\left(X_{\mu}\right)$ would acquire its mass through spontaneous symmetry breaking in the hidden sector. The scalar responsible for the phase transition can also thermalize the sterile sector in the early universe through Higgs' portal. The requirement of anomaly cancellation needs additional fermions in the spectrum that can be a dark matter candidate. However, for our analysis, we only focus on the sterile neutrino and its interactions.

The relevant part of the Lagrangian is the gauge interaction of the sterile neutrino, which is given by

$$
-\mathcal{L}_{s}=g_{X} \bar{\nu}_{s} \gamma^{\mu} P_{L} \nu_{s} X_{\mu}
$$

In terms of mass eigenstates,

$$
-\mathcal{L}_{s}=\sum_{i, j s} g_{i j} \bar{\nu}_{i} \gamma^{\mu} P_{L} \nu_{j} X_{\mu},
$$

where $g_{i j}=g_{X} U_{s i}^{*} U_{s j}$. The $4 \times 4$ Pontecorvo-MakiNakagawa-Sakata matrix is parametrized as

$$
U=R_{34} R_{24} R_{14} R_{23} R_{13} R_{12},
$$

where $R_{i j}$ is the rotation matrix in the $\mathrm{i}-\mathrm{j}$ plane. We assume that the elements of the mixing matrix are real as a contribution of the phases is negligible for the discussion that follows. We also fix the active neutrino mixing angles to the best-fit values from the oscillation measurements [36],

$$
\theta_{12}=33.62^{\circ} \quad \theta_{23}=47.2^{\circ} \quad \theta_{13}=8.54^{\circ} .
$$

We have six free parameters in our model,

$$
\mathcal{P}=\left\{\theta_{14}, \theta_{24}, \theta_{34}, m_{4}, g_{X}, M_{X}\right\},
$$

where $m_{4}$ is the mass of the fourth (mostly sterile) mass eigenstate and $M_{X}$ is the mass of the new gauge boson.

The introduction of self-interactions generates a finite temperature effective potential for the sterile neutrino of the form [10]

$$
V_{\text {eff }}= \begin{cases}-\frac{28 \pi^{3} \alpha_{X} E T_{s}^{4}}{45 M_{X}^{4}} & E, T_{s} \ll M \\ +\frac{\pi \alpha_{X} T_{s}^{2}}{2 E} & E, T_{s} \gg M,\end{cases}
$$

which modifies the effective mixing angle given by

$$
\sin ^{2}\left(2 \theta_{m}\right)=\frac{\sin ^{2}\left(2 \theta_{0}\right)}{\left(\cos \left(2 \theta_{0}\right)+\frac{2 E}{\Delta m^{2}} V_{\text {eff }}\right)^{2}+\sin ^{2}\left(2 \theta_{0}\right)} .
$$

In the early universe when the temperature is high, the mixing angle is suppressed and the production rate of the sterile neutrino is negligible. As the Universe cools, the sterile sector recouples to the standard model bath. If the recoupling temperature is $>\mathrm{MeV}$, then the sterile neutrinos are thermalized before the big bang nucleosynthesis takes place. Since they are relativistic during $\mathrm{BBN}$, there are very stringent constraints from Planck. Hence, one requires the recoupling temperature to be less than an $\mathrm{MeV}$. In [14] it was shown that the entire parameter space for the scenario is ruled out for $m_{4} \geq 1 \mathrm{eV}$. However, it was also pointed out that there are several possible new physics effects that can alleviate these bounds. One of the plausible scenarios is where one adds new lighter particles in the model.

\section{NEUTRINO ABSORPTION BY COSMIC NEUTRINO BACKGROUND}

Until very recently, the source of ultrahigh energy neutrinos was unknown. Advances in multimessenger astronomy have pointed towards blazars as possible sources 37]]. During propagation through the cosmic media, these neutrinos can get resonantly scattered off the cosmic neutrino background, which results in an absorption line in the neutrino spectrum. If only standard model interactions are considered, the absorption line $\left(\sim 10^{13} \mathrm{GeV}\right)$ is undetectable at neutrino telescopes [38]. However, it has been known that secret interaction of the neutrino can also give rise to these lines, which should, in principle, be detectable [39-41]. The absorption lines from sterile neutrino were first pointed out in [42], and the authors of [43] applied it in the context of diffuse supernova background. In this paper, we attempt to explain the two dips in the IceCube spectrum using resonant absorption by heavy (mostly) sterile neutrino and the heaviest active neutrino. 
We have assumed that, due to recoupling of the sterile neutrinos, the neutrino background has all four mass eigenstates in equal proportions and at the same temperature. For the benchmark scenarios considered in the paper, the recoupling is guaranteed [6]. The scattering cross section is

$\sigma_{i j}=\sigma\left(\bar{\nu}_{i} \nu_{j} \rightarrow \bar{\nu} \nu\right)=\frac{1}{6 \pi}\left|g_{i j}\right|^{2} g_{X}^{2} \frac{s}{\left(s-m_{X}^{2}\right)^{2}+m_{X}^{2} \Gamma_{X}^{2}}$,

where $\nu_{i}$ are the mass eigenstates of the four neutrino species and $\Gamma_{X}=g_{X}^{2} m_{X} / 12 \pi$ is the decay width of the new boson. The mean free path is

$$
\begin{aligned}
\lambda_{i}\left(E_{i}, z\right) & =\left(\sum_{j} \int \frac{d^{3} \mathbf{p}}{(2 \pi)^{3}} f_{j}(p, z) \sigma_{i j}\left(p, E_{i}, z\right)\right)^{-1} \\
& \approx\left(n_{\nu}(z) \sum_{j} \sigma_{i j}\left(p, E_{i}, z\right)\right)^{-1}
\end{aligned}
$$

where $f_{i}$ is the distribution function for the neutrinos given by

$$
f_{i}(p, z)^{-1}=\exp \left(\frac{p}{T_{i}(1+z)}\right)+1
$$

and $T_{i}=1.95 \mathrm{~K}$ for all four components. The approximation in the rhs of Eq. (10) is valid only when the neutrino is nonrelativistic. The oscillation data suggest that at least two active neutrinos are nonrelativistic today. As we see, the lightest neutrino gives the absorption feature for higher energies and is inconsequential to our discussion. For the remainder of the paper, we assume normal hierarchy and neutrino masses to be

$$
\begin{aligned}
& m_{1}=5 \times 10^{-3} \mathrm{eV}, \quad m_{2}=1 \times 10^{-2} \mathrm{eV}, \\
& m_{3}=5 \times 10^{-2} \mathrm{eV} .
\end{aligned}
$$

The case of inverted hierarchy is commented upon at the end of this section. One can see that

$$
m_{i} \gg\langle p\rangle=3 T_{\nu} \sim 5.3 \times 10^{-4} \mathrm{eV} \quad \forall i,
$$

which allows us to approximate

$$
s=2 E_{i}(1+z)\left(\sqrt{p^{2}+m_{i}^{2}}-p \cos [\theta]\right) \approx 2 E_{i}(1+z) m_{i} .
$$

The $z$ dependence accounts for redshift during propagation. The survival rate of the neutrino is given as $[41,44]$

$$
R_{i}=\exp \left[-\int_{0}^{z_{s}} \frac{1}{\lambda_{i}(1+z)} \frac{d L}{d z} d z\right]
$$

where $z_{s}$ denotes the redshift distance to the source and

$$
\frac{d L}{d z}=\frac{c}{H_{0} \sqrt{\Omega_{m}(1+z)^{3}+\Omega_{\Lambda}}} .
$$

We have fixed the cosmological parameters to $\Omega_{m}=0.315$, $\Omega_{\Lambda}=0.685, H_{0}=67.3 \mathrm{~km} / \mathrm{s} / \mathrm{Mpc}$ using the best-fit values from Planck [4]. We also assume a power-law flux for each neutrino near the source. The flux of neutrino of flavor $\alpha \in e, \mu, \tau, s$ at Earth is

$\phi_{\alpha}=\sum_{j=1}^{4}\left|U_{\alpha j}\right|^{2} \phi_{j} R_{j}=\left(\phi_{0} E_{\nu}^{-\gamma}\right) \sum_{j=1}^{4}\left|U_{\alpha j}\right|^{2} R_{j} \equiv\left(\phi_{0} E_{\nu}^{-\gamma}\right) R_{\alpha}$.

Since the sterile neutrino does not generate any signal at the IceCube detector, the flux of neutrinos that can be seen by IceCube is simply

$$
\begin{aligned}
\phi & =\phi_{e}+\phi_{\mu}+\phi_{\tau} \\
& =\left(\phi_{0} E_{\nu}^{-\gamma}\right)\left(\sum_{f=e, \mu, \tau} \sum_{j=1}^{4}\left|U_{f j}\right|^{2} R_{j}\right) \\
& \equiv \phi_{0} E_{\nu}^{-\gamma}\left\langle R\left(\mathcal{P}, E_{\nu}\right)\right\rangle,
\end{aligned}
$$

where the parentheses in the last part indicate that $\langle R\rangle$ depends on the model parameters and incident neutrino energy only.

In Fig. 1, we have shown the variation of $R_{\alpha}$ and $R_{i}$ for a benchmark scenario. The gauge coupling is fixed to be $g_{X}=0.1$ and the mass of the gauge boson is fixed to be $M_{X}=25 \mathrm{MeV}$. We have assumed that the neutrino sources are localized around $z_{s}=0.3$. There are three features we would like to highlight: (a) There are two prominent dips in the function. The one at lower neutrino energy is associated with the absorption due to heavy (i.e., mostly sterile) mass eigenstate. The second dip is due to the absorption by the heaviest active neutrino (i.e., $m_{3}$ in NH). (b) The dips are not very sharp and there is a broadening due to redshift during propagation. For a source located at $z_{s}$, the dip in the spectrum occurs for the neutrino energies

$$
E_{\text {dip }}: \frac{E^{\text {res }}}{\left(1+z_{s}\right)} \rightarrow E^{\text {res }}
$$

where $E^{\text {res }}=M_{X}^{2} / 2 m_{i}$. This allows us to estimate the width of the dip as

$$
\Delta^{i} \approx \frac{M_{X}^{2}}{2 m_{i}} \frac{z_{s}}{1+z_{s}}
$$

(c) Since the other active neutrinos are lighter, their absorption lines are at much higher neutrino energies. Hence, it is inconsequential for our analysis whether the lightest neutrino is relativistic or nonrelativistic today. 

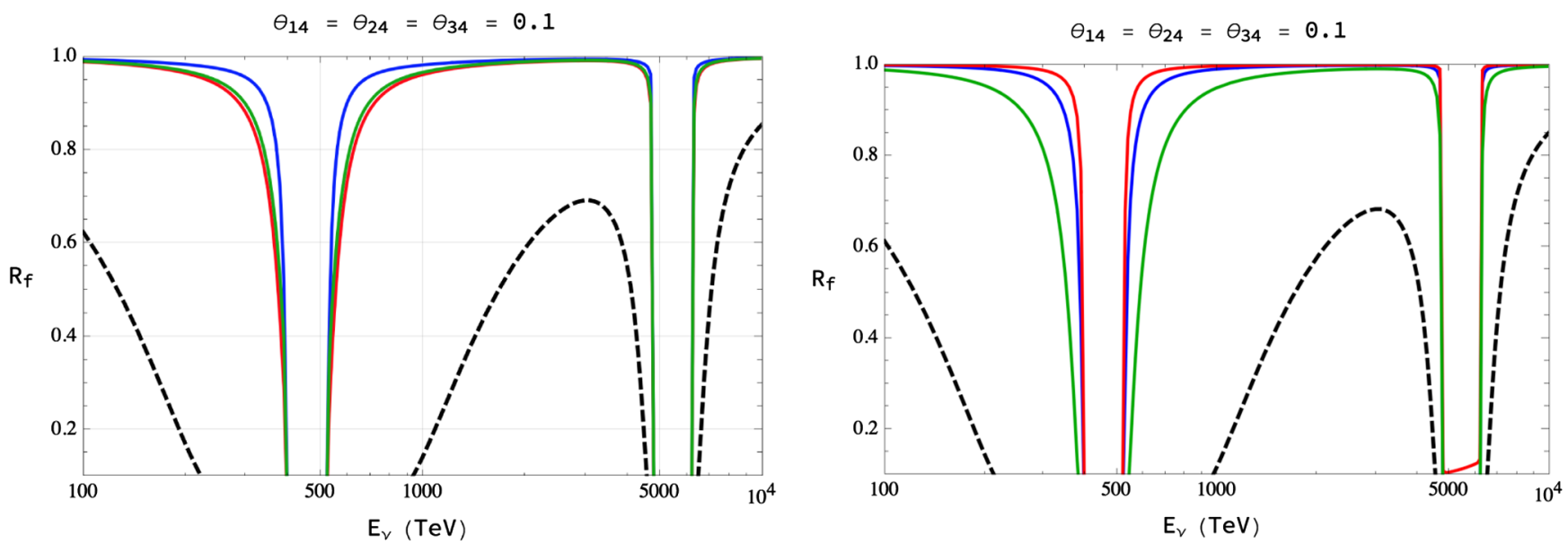

FIG. 1. Left: This plot shows variation of $R_{e}$ (blue), $R_{\mu}$ (red), $R_{\tau}$ (green), and $R_{s}$ (black, dashed) with neutrino energy. Right: This plot shows variation of $R_{1}$ (blue), $R_{2}$ (red), $R_{3}$ (green), and $R_{4}$ (black, dashed) with neutrino energy. See text for details.

The absorption lines are sensitive to the distance to the source. It can be inferred from (20) that the further the source, the broader the absorption line. We have assumed that the ultra-high energy (UHE) neutrinos originate from blazars and non-blazar active galactic nuclei as opposed to spatially distributed sources like dark matter decay [37,4548]. Future multimessenger observations will help us verify this hypothesis. For this analysis we assume that the sources are localized around a particular redshift, $\left\langle z_{s}\right\rangle$, which makes the calculations simple. The complete analysis that also considers distribution of the sources is beyond the scope of this work. Also note that any source located very far from Earth $\left(z_{s}>5\right)$ will have too broad absorption lines and contribute negligibly to the flux at high energies $(>200 \mathrm{TeV}$ ). This may be compatible with the fact that IceCube rarely sees events of such high energies. This inference cannot be made in the standard picture without secret interactions. Thus, if future multimessenger observations infer that almost all the sources of UHE neutrinos are localized within a sphere, it will strongly hint at resonant absorption.

\section{CONSTRAINTS FROM FLUX OF NEUTRINOS AT ICECUBE}

In IceCube six-year HESE data, 82 events passed the selection criterion of which two are co-incident with atmospheric muons and left out [20]. The best fit for single power-law flux is

$$
\begin{aligned}
E_{\nu}^{2} \phi= & (2.46 \pm 0.8) \\
& \times 10^{-8}\left(\frac{E_{\nu}}{100 \mathrm{TeV}}\right)^{-0.92} \mathrm{GeV} \mathrm{cm}^{-2} \mathrm{~s}^{-1} \mathrm{sr}^{-1},
\end{aligned}
$$

which has a softer spectral index than the 3-year $(\gamma=2.3)$ [18] as well as the 4-year $(\gamma=2.58)$ data [19]. One can attribute this to the pileup of low energy events along with the lack of high energy events in the new data. A prominent feature that still remains is the apparent lack of neutrinos with energy 400-800 TeV. From one point of view, one should be able to see these neutrinos with more exposure. However, this may also hint at new physics. Another puzzling mystery is the absence of Glashow resonance. In the standard model, the astrophysical neutrino can interact with the electrons in the detector volume and produce an on-shell W-boson. This happens for neutrino energy $\sim 6.3 \mathrm{PeV}$. Around this energy, the cross section for neutrino-electron scattering is several orders of magnitude larger than the charged and neutral current interactions with nucleons. Thus we expect more numbers of events in the 3.6 to $7.5 \mathrm{PeV}$ bin. Because of this, the best fits to the data hint towards a softer spectral index. Several scenarios have been proposed to address the absence of Glashow events including active neutrino decay, $\Delta^{+}$resonance, and novel flux [49-51].

Now we examine the $m_{s}-M_{X}$ parameter space that can explain the observed IceCube spectrum. The following constraints are imposed.

(1) If $E^{\text {res }} \sim \mathrm{PeV}$, one cannot explain the observed $\mathrm{PeV}$ events at IceCube unless exceptional circumstances are evoked. To be general, we constrain the $m_{3}$ absorption line to be more than $3 \mathrm{PeV}$. Because of the broadening during propagation, the constraint depends on $\left\langle z_{s}\right\rangle$ as

$$
M_{X}^{2} \geq 2 \times 3 \mathrm{PeV} m_{3}\left(1+\left\langle z_{s}\right\rangle\right)
$$

This is shown in Fig. 2 as a region bounded by green lines.

(2) Since we wish to explain the dip in the spectrum using the fourth neutrino, we require 


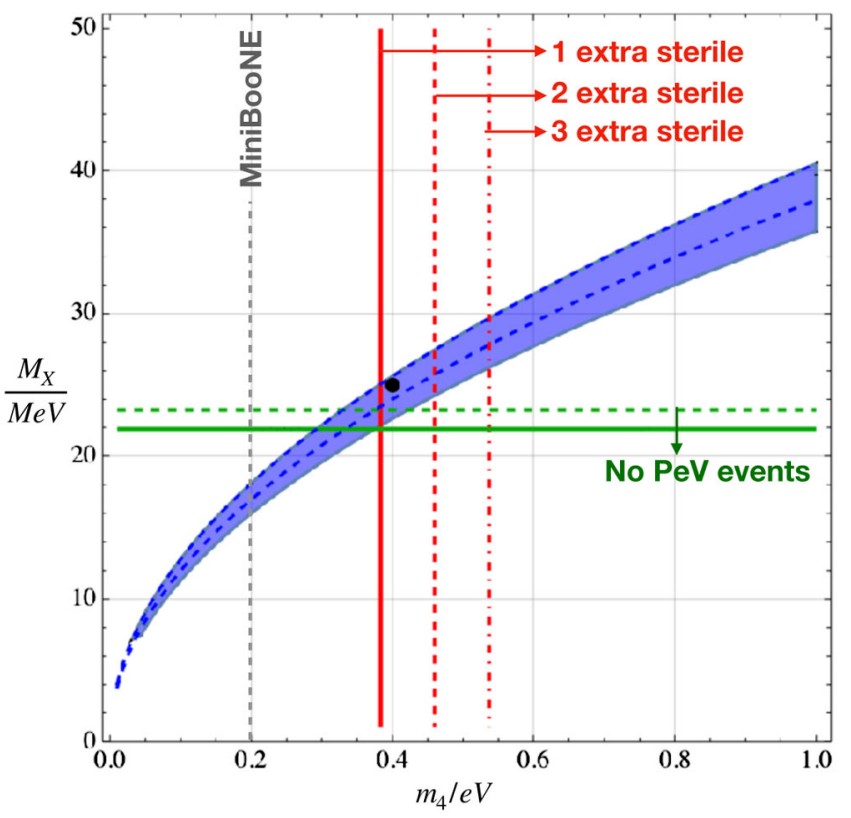

FIG. 2. The shaded blue region with solid (dashed) boundaries can explain the 400-800 $\mathrm{TeV}$ dip in the IceCube spectrum assuming that the sources are distributed around $\mathrm{z}=0.6(0.8)$. The solid (dashed) green lines denote the upper bound on the $\mathrm{X}$ boson mass such that the gap due to heaviest active neutrino is above $3 \mathrm{PeV}$ assuming source distribution around $\mathrm{z}=0.6(0.8)$. The green arrows indicate the region that is disfavored. The red lines (solid, dashed, dot-dashed) denote the number of additional light particles $(1,2,3)$ to be added to the theory to evade $\sum m_{\nu}$ constraints. The black point shows the benchmark case considered in the paper. The MiniBooNE best fit is highlighted. See the text for more details.

$$
E^{\mathrm{res}} \leq 800 \mathrm{TeV} \quad \& \quad \frac{E^{\mathrm{res}}}{1+\left\langle z_{s}\right\rangle} \geq 400 \mathrm{TeV},
$$

which is shown as the blue shaded region in Fig. 2.

(3) We show the region in the parameter space that requires more than 1,2 , and 3 lighter sterile neutrinos in the full theory [cf., Eq. (10)].

It can be seen from Fig. 2 that only a small portion of the parameter space is compatible with all the constraints. With slightly relaxed assumptions, we chose the representative point

$$
m_{4}=0.4 \mathrm{eV} \quad \& \quad M_{X}=25 \mathrm{MeV}
$$

for our analysis. The gauge coupling is constrained from the restrictions on the recoupling temperature. We have chosen the benchmark point $g_{X}=0.1$, which is consistent.

For the choice of mixing angles, we have considered two scenarios,

Case I: $\theta_{14}=\theta_{24}=\theta_{34}=0.3 \quad \ldots($ democratic $)$,

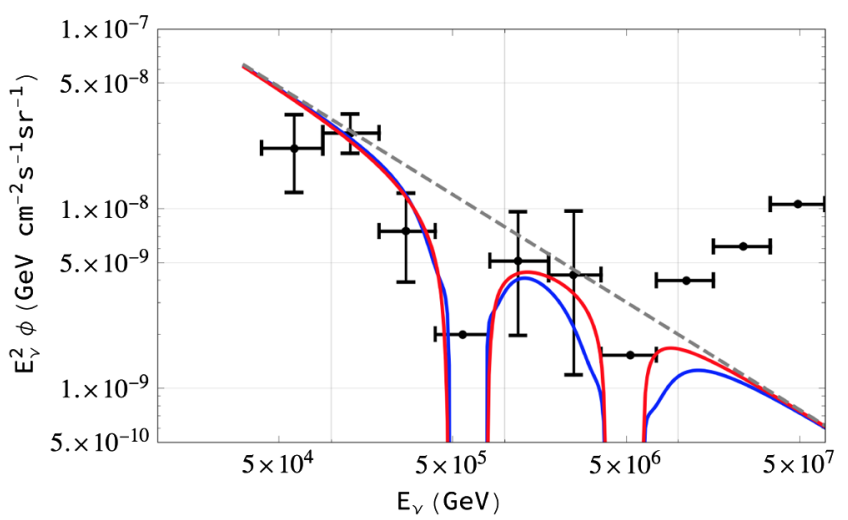

FIG. 3. The flux without attenuation is shown as the dashed gray curve. The blue (red) curve is the flux with attenuation for the democratic (maximal) case. The spectral index is chosen to be 2.6 and the normalization is fixed from the second bin. Sources are assumed to be distributed around $\mathrm{z}=0.6$.

Case II: $\theta_{14}=\theta_{24}=\pi / 4 \quad \& \quad \theta_{34}=0 \quad \ldots$ (maximal).

For the democratic case, we have checked that the choice 0.3 gives the best fit to the data. The maximal case is motivated by the mixing angles observed by MiniBooNE. We have chosen the spectral index to be 2.6 , which is consistent with IceCube best fits. Any softer spectral index will result in reducing the flux of $\mathrm{PeV}$ neutrinos, which is unwanted. For harder spectral index, one needs to assume larger values of $\left\langle z_{s}\right\rangle$ to be compatible. The attenuated flux is shown in Fig. 3.

\section{CONCLUSION}

To reconcile a light sterile neutrino of the type observed by MiniBooNE with BBN predictions, one must introduce gauge or scalar mediated interactions between the sterile neutrinos. Because of the lightness of the mediators required, there are observable effects in the spectrum of high energy neutrinos detected by IceCube. We have shown that the gaps in the spectrum at $400-800 \mathrm{TeV}$ as well as beyond 2.6 PeV correspond to resonant absorption of two heaviest mass eigenstates. The prediction for the model at IceCube is peaks beyond $6.3 \mathrm{PeV}$ and dips corresponding to two lighter neutrino mass states. These features may be observable in future IceCube data. A generic feature of absorption during propagation is that energy gap in the spectrum widens with distance to the source. This renders IceCube invisible to $\nu$ sources beyond a certain $z_{\max }$. Future multimessenger observations should be able to confirm this. 
[1] A. A. Aguilar-Arevalo et al. (MiniBooNE Collaboration), arXiv:1805.12028.

[2] A. A. Aguilar-Arevalo et al. (LSND Collaboration), Phys. Rev. D 64, 112007 (2001).

[3] M. Dentler, Á. Hernndez-Cabezudo, J. Kopp, P. Machado, M. Maltoni, I. Martinez-Soler, and T. Schwetz, J. High Energy Phys. 08 (2018) 010.

[4] N. Aghanim et al. (Planck Collaboration), arXiv:1807 .06209 .

[5] S. Hannestad, R. S. Hansen, and T. Tram, Phys. Rev. Lett. 112, 031802 (2014).

[6] X. Chu, B. Dasgupta, and J. Kopp, J. Cosmol. Astropart. Phys. 10 (2015) 011.

[7] M. Archidiacono, S. Hannestad, R. S. Hansen, and T. Tram, Phys. Rev. D 91, 065021 (2015).

[8] M. Archidiacono, S. Hannestad, R. S. Hansen, and T. Tram, Phys. Rev. D 93, 045004 (2016).

[9] M. Archidiacono, S. Gariazzo, C. Giunti, S. Hannestad, R. Hansen, M. Laveder, and T. Tram, J. Cosmol. Astropart. Phys. 08 (2016) 067.

[10] B. Dasgupta and J. Kopp, Phys. Rev. Lett. 112, 031803 (2014).

[11] N. Saviano, O. Pisanti, G. Mangano, and A. Mirizzi, Phys. Rev. D 90, 113009 (2014).

[12] A. Mirizzi, G. Mangano, O. Pisanti, and N. Saviano, Phys. Rev. D 91, 025019 (2015).

[13] F. Forastieri, M. Lattanzi, G. Mangano, A. Mirizzi, P. Natoli, and N. Saviano, J. Cosmol. Astropart. Phys. 07 (2017) 038.

[14] X. Chu, B. Dasgupta, M. Dentler, J. Kopp, and N. Saviano, arXiv:1806.10629.

[15] B. Chauhan, Phys. Rev. D 97, 123017 (2018).

[16] T. Bringmann, J. Hasenkamp, and J. Kersten, J. Cosmol. Astropart. Phys. 07 (2014) 042.

[17] M. Ibe and K. Kaneta, Phys. Rev. D 90, 053011 (2014).

[18] M. G. Aartsen et al. (IceCube Collaboration), Phys. Rev. Lett. 113, 101101 (2014).

[19] M. G. Aartsen et al. (IceCube Collaboration), arXiv:1510 .05223 .

[20] D. Williams (IceCube Collaboration), Int. J. Mod. Phys. Conf. Ser. 46, 1860048 (2018).

[21] T. Araki, F. Kaneko, Y. Konishi, T. Ota, J. Sato, and T. Shimomura, Phys. Rev. D 91, 037301 (2015).

[22] S. Mohanty, A. Narang, and S. Sadhukhan, arXiv:1808 .01272 .

[23] A. Bhattacharya, R. Gandhi, A. Gupta, and S. Mukhopadhyay, J. Cosmol. Astropart. Phys. 05 (2017) 002.

[24] A. Bhattacharya, A. Esmaili, S. Palomares-Ruiz, and I. Sarcevic, J. Cosmol. Astropart. Phys. 07 (2017) 027.

[25] M. Dhuria and V. Rentala, J. High Energy Phys. 09 (2018) 004.
[26] C. Rott, K. Kohri, and S. C. Park, Phys. Rev. D 92, 023529 (2015).

[27] P. S. B. Dev, D. Kazanas, R. N. Mohapatra, V. L. Teplitz, and Y. Zhang, J. Cosmol. Astropart. Phys. 08 (2016) 034.

[28] Y. Sui and P. S. Bhupal Dev, J. Cosmol. Astropart. Phys. 07 (2018) 020.

[29] L. A. Anchordoqui, C. A. Garcia Canal, H. Goldberg, D. Gomez Dumm, and F. Halzen, Phys. Rev. D 74, 125021 (2006).

[30] V. Barger and W. Y. Keung, Phys. Lett. B 727, 190 (2013).

[31] P. S. B. Dev, D. K. Ghosh, and W. Rodejohann, Phys. Lett. B 762, 116 (2016).

[32] U. K. Dey and S. Mohanty, J. High Energy Phys. 04 (2016) 187.

[33] N. Mileo, A. de la Puente, and A. Szynkman, J. High Energy Phys. 11 (2016) 124.

[34] B. Chauhan, B. Kindra, and A. Narang, Phys. Rev. D 97, 095007 (2018).

[35] U. K. Dey, D. Kar, M. Mitra, M. Spannowsky, and A. C. Vincent, Phys. Rev. D 98, 035014 (2018).

[36] I. Esteban, M. C. Gonzalez-Garcia, M. Maltoni, I. MartinezSoler, and T. Schwetz, J. High Energy Phys. 01 (2017) 087.

[37] M. G. Aartsen et al. (IceCube, Fermi-LAT, MAGIC, AGILE, ASAS-SN, HAWC, H.E.S.S., INTEGRAL, Kanata, Kiso, Kapteyn, Liverpool Telescope, Subaru, Swift NuSTAR, VERITAS, and VLA/17B-403 Collaborations) Science 361, eaat1378 (2018).

[38] T. J. Weiler, Phys. Rev. Lett. 49, 234 (1982).

[39] K. C. Y. Ng and J. F. Beacom, Phys. Rev. D 90, 065035 (2014); 90, 089904(E) (2014).

[40] K. Ioka and K. Murase, Prog. Theor. Exp. Phys. 2014, 61E01 (2014).

[41] K. Blum, A. Hook, and K. Murase, arXiv:1408.3799.

[42] J. F. Cherry, A. Friedland, and I. M. Shoemaker, arXiv:1605 .06506.

[43] Y. S. Jeong, S. Palomares-Ruiz, M. H. Reno, and I. Sarcevic, J. Cosmol. Astropart. Phys. 06 (2018) 019.

[44] A. DiFranzo and D. Hooper, Phys. Rev. D 92, 095007 (2015).

[45] F. Krau et al., Astron. Astrophys. 566, L7 (2014).

[46] S. Sahu, L. S. Miranda, and A. Rosales De Len, Proc. Sci. NEUTEL2017 (2018) 079.

[47] L. S. Miranda, A. R. de Len, and S. Sahu, Eur. Phys. J. C 76, 402 (2016).

[48] D. Hooper, T. Linden, and A. Vieregg, arXiv:1810.02823.

[49] S. Pakvasa, A. Joshipura, and S. Mohanty, Phys. Rev. Lett. 110, 171802 (2013).

[50] S. Sahu and B. Zhang, J. High Energy Astrophys. 18, 1 (2018).

[51] M. D. Kistler and R. Laha, Phys. Rev. Lett. 120, 241105 (2018). 